\title{
HERMITE-HADAMARD TYPE INEQUALITIES FOR SOME CONVEX DOMINATED FUNCTIONS VIA FRACTIONAL INTEGRALS
}

\author{
HAVVA KAVURMACI-ÖNALAN \\ Received 12 September, 2020
}

\begin{abstract}
In this study, we derive some new inequalities of H-H type for $(g, m)$ - and $(g, h)$ convex dominated functions related fractional integral. Our obtained results are extensions of earlier works.
\end{abstract}

2010 Mathematics Subject Classification: 05C38; 26D10; 26D15

Keywords: Convex Dominated Functions, H-H Inequality, Fractional Calculus

\section{INTRODUCTION}

Let's start our study by introducing the concept of convex function and the famous inequality obtained for the mean value of a convex function (see [6], [15]).

Definition 1. Let $I \subseteq \mathbb{R}$ be an interval. Then a real-valued function $f: I \rightarrow \mathbb{R}$ is said to be convex (concave) on the interval $I$ if the inequality

$$
f(\lambda x+(1-\lambda) y) \leq(\geq) \lambda f(x)+(1-\lambda) f(y)
$$

holds for all $x, y \in I$ and $\lambda \in[0,1]$.

With the help of convex function and inequalities we will give below, we observe that many applications take place in pure and applied mathematics.

The following double inequality is called Hermite-Hadamard inequality in the literature. If $f: I \rightarrow \mathbb{R}$ is a convex function on the interval $I$ of real numbers and $a, b \in I$ with $a<b$, then

$$
f\left(\frac{a+b}{2}\right) \leq \frac{1}{b-a} \int_{a}^{b} f(x) d x \leq \frac{f(a)+f(b)}{2} .
$$

The inequalities in (1.2) hold in the reversed direction if $f$ is concave.

Different convex function types and different Hermite-Hadamard type inequalities which are considered basic for each definition and for each inequality have been 
obtained through the Definition 1 and Hermite-Hadamard Inequality. It is observed that studies in this direction take a large place in the literature.

In [22], G. Toader defined $m$-convexity as the following:

Definition 2. The function $f:[0, b] \rightarrow \mathbb{R}, b>0$ is said to be $m$-convex where $m \in[0,1]$, if we have

$$
f(\lambda x+m(1-\lambda) y) \leq \lambda f(x)+m(1-\lambda) f(y)
$$

for all $x, y \in[0, b]$ and $\lambda \in[0,1]$. We say that $f$ is $m$-concave if $(-f)$ is $m$-convex.

In [4], Dragomir obtained the following Theorem for $m$ - convex functions.

Theorem 1. Let $f:[0, \infty) \rightarrow \mathbb{R}$ be a $m$-convex function with $m \in(0,1]$ and $0 \leq a<b$. If $f \in L_{1}[a, b]$, then one has the inequalities

$$
\begin{aligned}
f\left(\frac{a+b}{2}\right) & \leq \frac{1}{b-a} \int_{a}^{b} \frac{f(x)+m f\left(\frac{x}{m}\right)}{2} d x \\
& \leq \frac{1}{2}\left[\frac{f(a)+m f\left(\frac{a}{m}\right)}{2}+m \frac{f\left(\frac{b}{m}\right)+m f\left(\frac{b}{m^{2}}\right)}{2}\right] .
\end{aligned}
$$

Let us remind the definition of $h$ - convex function [11,23]:

Definition 3. Let $h-$ be a positive function. We say that $f: I \subseteq \mathbb{R} \rightarrow \mathbb{R}$ is $h$-convex function or that belongs to the class $S X(h, I)$, if $f$ is non-negative and for all $x, y \in I$ and $\lambda \in(0,1)$, we have

$$
f(\lambda x+(1-\lambda) y) \leq h(\lambda) f(x)+h(1-\lambda) f(y) .
$$

If the inequality in (1.4) is reserved, then $f$ is said to be $h$-concave, i.e. $S V(h, I)$. Obviously, if $f(\lambda)=\lambda ; f(\lambda)=\frac{1}{\lambda} ; f(\lambda)=1 ; f(\lambda)=\lambda^{s}$ where $s \in(0,1)$, then all nonnegative convex function belong to $S X(h, I)$ and all nonnegative concave functions belong to $S V(h, I) ; S X(h, I)=Q(I) ; S X(h, I) \supseteq P(I) ; S X(h, I) \supseteq K_{s}^{2}$, respectively.

The classical H-H inequality for $h-$ convex functions was obtained by Sarikaya et. al. in [18] is as follows:

Theorem 2. Let $f \in S X(h, I), a, b \in I$ with $a<b, f \in L_{1}[a, b]$. Then

$$
\frac{1}{2 h\left(\frac{1}{2}\right)} f\left(\frac{a+b}{2}\right) \leq \frac{1}{b-a} \int_{a}^{b} f(x) d x \leq[f(a)+f(b)] \int_{0}^{1} h(\lambda) d \lambda .
$$

In [5], Dragomir and Ionescu introduced the following class of functions and proved some inequalities.

Definition 4. Let $g: I \rightarrow \mathbb{R}$ be a convex function on the interval $I$. The function $g: I \rightarrow R$ is called $g$-convex dominated on $I$ if the following condition is satisfied: $|\lambda f(x)+(1-\lambda) f(y)-f(\lambda x+(1-\lambda) y)| \leq \lambda g(x)+(1-\lambda) g(y)-g(\lambda x+(1-\lambda) y)$ for all $x, y \in I$ and $\lambda \in[0,1]$. 
Theorem 3. (See [7]) Let $g: I \rightarrow \mathbb{R}$ be a convex function and $f: I \rightarrow R$ be a $g-$ convex dominated mapping. Then, for all $a, b \in I, a<b$,

$$
\left|f\left(\frac{a+b}{2}\right)-\frac{1}{b-a} \int_{a}^{b} f(x) d x\right| \leq \frac{1}{b-a} \int_{a}^{b} g(x) d x-g\left(\frac{a+b}{2}\right)
$$

and

$$
\left|\frac{f(a)+f(b)}{2}-\frac{1}{b-a} \int_{a}^{b} f(x) d x\right| \leq \frac{g(a)+g(b)}{2}-\frac{1}{b-a} \int_{a}^{b} g(x) d x .
$$

$(g, m)$ - dominated convex function and interested theorem have been given as the following (see [12]):

Definition 5. Let $g:[0, b] \rightarrow \mathbb{R}$ be a given $m$ - convex function on the interval $[0, b]$. The real function $f:[0, b] \rightarrow \mathbb{R}$ is called $(g, m)$-convex dominated on $[0, b]$ if the following condition is satisfied

$$
\begin{aligned}
& |\lambda f(x)+m(1-\lambda) f(y)-f(\lambda x+m(1-\lambda) y)| \\
& \quad \leq \lambda g(x)+m(1-\lambda) g(y)-g(\lambda x+m(1-\lambda) y)
\end{aligned}
$$

for all $x, y \in[0, b], m, \lambda \in[0,1]$.

Theorem 4. Let $g:[0, \infty) \rightarrow \mathbb{R}$ be an $m$-convex function with $m \in(0,1] . \quad f$ : $[0, \infty) \rightarrow \mathbb{R}$ is $(g, m)$ - convex dominated mapping and $0 \leq a<b$. If $f \in L_{1}[a, b]$, then one has the inequalities:

$$
\begin{aligned}
& \left|\frac{1}{b-a} \int_{a}^{b} \frac{f(x)+m f\left(\frac{x}{m}\right)}{2} d x-f\left(\frac{a+b}{2}\right)\right| \\
& \quad \leq \frac{1}{b-a} \int_{a}^{b} \frac{g(x)+m g\left(\frac{x}{m}\right)}{2} d x-g\left(\frac{a+b}{2}\right)
\end{aligned}
$$

and

$$
\begin{aligned}
& \left|\frac{1}{2}\left[\frac{f(a)+m f\left(\frac{a}{m}\right)}{2}+m \frac{f\left(\frac{b}{m}\right)+m f\left(\frac{b}{m^{2}}\right)}{2}\right]-\frac{1}{b-a} \int_{a}^{b} \frac{f(x)+m f\left(\frac{x}{m}\right)}{2} d x\right| \\
& \quad \leq \frac{1}{2}\left[\frac{g(a)+m g\left(\frac{a}{m}\right)}{2}+m \frac{g\left(\frac{b}{m}\right)+m g\left(\frac{b}{m^{2}}\right)}{2}\right]-\frac{1}{b-a} \int_{a}^{b} \frac{g(x)+m g\left(\frac{x}{m}\right)}{2} d x .
\end{aligned}
$$

Definition 6. (See [12]) Let $h \neq 0, h: J \rightarrow \mathbb{R}$ be a nonnegative function, $g: I \rightarrow$ $\mathbb{R}$ be an $h$-convex function. The real function $f: I \rightarrow \mathbb{R}$ is called $(g, h)$-convex dominated on $I$ if the following condition is satisfied:

$$
\begin{aligned}
& |h(\lambda) f(x)+h(1-\lambda) f(y)-f(\lambda x+(1-\lambda) y)| \\
& \quad \leq h(\lambda) g(x)+h(1-\lambda) g(y)-g(\lambda x+(1-\lambda) y)
\end{aligned}
$$

for all $x, y \in I$ and $\lambda \in(0,1]$. 
Theorem 5. Let $h: J \rightarrow \mathbb{R}$ be a non-negative function, $h \neq 0, g: I \rightarrow \mathbb{R}$, be an $h-$ convex function and the real function $f: I \rightarrow \mathbb{R}$ be $(g, h)$ - convex dominated on $I$. Then one has the inequalities:

$$
\begin{aligned}
& \left|\frac{1}{b-a} \int_{a}^{b} f(x) d x-\frac{1}{2 h\left(\frac{1}{2}\right)} f\left(\frac{a+b}{2}\right)\right| \\
& \quad \leq \frac{1}{b-a} \int_{a}^{b} g(x) d x-\frac{1}{2 h\left(\frac{1}{2}\right)} g\left(\frac{a+b}{2}\right)
\end{aligned}
$$

and

$$
\begin{aligned}
& \left|[f(a)+f(b)] \int_{0}^{1} h(\lambda) d \lambda-\frac{1}{b-a} \int_{a}^{b} f(x) d x\right| \\
& \leq[g(a)+g(b)] \int_{0}^{1} h(\lambda) d \lambda-\frac{1}{b-a} \int_{a}^{b} g(x) d x
\end{aligned}
$$

for all $x, y \in I$ and $\lambda \in(0,1]$.

Many authors study integral inequalities involving various fractional operators like Erdelyi-Kober, Riemann-Liouville, conformable fractional integral operators, Katugampola, etc. in last years. The most studied in them is Riemann-Liouville fractional integral operators. In [16], Liouville and Riemann introduced the fractional calculus at last of the nineteenth century. Now, we remind the definition of Riemann-Liouville fractional integrals.

Definition 7. Let $f \in L_{1}[a, b]$. The Riemann-Liouville integrals $J_{a^{+}}^{\alpha} f$ and $J_{b^{-}}^{\alpha} f$ of order $\alpha>0$ with $a \geq 0$ are defined by

$$
J_{a^{+}}^{\alpha} f(x)=\frac{1}{\Gamma(\alpha)} \int_{a}^{x}(x-t)^{\alpha-1} f(t) d t, x>a
$$

and

$$
J_{b^{-}}^{\alpha} f(x)=\frac{1}{\Gamma(\alpha)} \int_{x}^{b}(t-x)^{\alpha-1} f(t) d t, x<b
$$

where $\Gamma(\alpha)=\int_{0}^{\infty} e^{-t} u^{\alpha-1} d u$. Here $J_{a^{+}}^{0} f(x)=J_{b^{-}}^{0} f(x)=f(x)$. In the case of $\alpha=1$, the fractional integral reduces to the classical integral.

One can find the interested properties and inequalities the references $[1-3,8-10$, 13, 16, 17, 19-21, 24, 25].

In [19], Sarikaya et. al. obtained the Hermite-Hadamard type inequality for fractional calculus as following:

Theorem 6. Let $f:[a, b] \rightarrow \mathbb{R}$ be positive function with $0 \leq a<b$ and $f \in L_{1}[a, b]$. If $f$ is a convex function on $[a, b]$, then the following inequalities for fractional integrals hold:

$$
f\left(\frac{a+b}{2}\right) \leq \frac{\Gamma(\alpha+1)}{2(b-a)^{\alpha}}\left[J_{a^{+}}^{\alpha} f(b)+J_{b^{-}}^{\alpha} f(a)\right] \leq \frac{f(a)+f(b)}{2}
$$


with $\alpha \geq 0$.

In [14], another Hermite-Hadamard type inequality via fractional calculus have been presented by Özdemir and Önalan.

Theorem 7. Let $f, g:[a, b] \rightarrow \mathbb{R}$ be positive functions with $0 \leq a<b$ and $f, g \in$ $L_{1}[a, b]$. If $g$ is a convex function on $[a, b]$ and $f$ is a $g$ - convex dominated function, then the following inequalities for fractional integrals hold:

$$
\begin{aligned}
& \left|\frac{\Gamma(\alpha+1)}{2(b-a)^{\alpha}}\left[J_{a^{+}}^{\alpha} f(b)+J_{b^{-}}^{\alpha} f(a)\right]-f\left(\frac{a+b}{2}\right)\right| \\
& \quad \leq \frac{\Gamma(\alpha+1)}{2(b-a)^{\alpha}}\left[J_{a^{+}}^{\alpha} g(b)+J_{b^{-}}^{\alpha} g(a)\right]-g\left(\frac{a+b}{2}\right)
\end{aligned}
$$

and

$$
\begin{aligned}
& \left|\frac{f(a)+f(b)}{2}-\frac{\Gamma(\alpha+1)}{2(b-a)^{\alpha}}\left[J_{a^{+}}^{\alpha} f(b)+J_{b^{-}}^{\alpha} f(a)\right]\right| \\
& \quad \leq \frac{g(a)+g(b)}{2}-\frac{\Gamma(\alpha+1)}{2(b-a)^{\alpha}}\left[J_{a^{+}}^{\alpha} g(b)+J_{b^{-}}^{\alpha} g(a)\right] .
\end{aligned}
$$

In [14], Özdemir and Önalan present Hermite-Hadamard type inequalities for fractional calculus as following:

Theorem 8. Let $f:[0, \infty) \rightarrow \mathbb{R}$ be a positive function with $0 \leq a<b$ and $f \in$ $L_{1}\left[a, \frac{b}{m}\right]$. If $f$ is an m-convex function on $[0, \infty)$, then the following inequality for fractional integrals holds:

$$
\begin{aligned}
f\left(\frac{a+b}{2}\right) & \leq \frac{\Gamma(\alpha+1)}{2(b-a)^{\alpha}}\left[J_{a^{+}}^{\alpha} f(b)+m^{\alpha+1} J_{\frac{b}{m}}^{\alpha} f\left(\frac{a}{m}\right)\right] \\
& \leq \frac{1}{2}\left[\frac{\alpha f(a)+m f\left(\frac{a}{m}\right)}{\alpha+1}+m \frac{f\left(\frac{b}{m}\right)+m \alpha f\left(\frac{b}{m^{2}}\right)}{\alpha+1}\right]
\end{aligned}
$$

with $\alpha>0$ ve $m \in(0,1]$.

Yildiz et. al give Hermite-Hadamard type inequality for fractional calculus in [25].

Theorem 9. Let $f: I \subseteq R \rightarrow \mathbb{R}$ be a real function with $a<b, a, b \in I^{\star}$ and $f \in$ $L[a, b]$. If $f$ belongs to the $S X(h, I)$, we give

$$
\begin{aligned}
\frac{1}{\alpha h\left(\frac{1}{2}\right)} f\left(\frac{a+b}{2}\right) & \leq \frac{\Gamma(\alpha)}{(b-a)^{\alpha}}\left[J_{a^{+}}^{\alpha} f(b)+J_{b^{-}}^{\alpha} f(a)\right] \\
& \leq[f[a]+f[b]] \int_{0}^{1} t^{\alpha-1}[h(t)+h(1-t)] d t
\end{aligned}
$$

with $\alpha>0$.

Now, we give new Hermite-Hadamard type inequalities for $(g, h)$-convex dominated functions and $(g, m)$-convex dominated functions by using fractional calculus. 


\section{THE RESULTS}

Theorem 10. Let $f, g:[a, b] \rightarrow \mathbb{R}$ be positive functions with $0 \leq a<b$ and $f, g \in$ $L_{1}[a, b]$. If $g$ is an $h$-convex function on $[a, b]$ and $f$ is a $(g, h)$ - convex dominated function, then the following inequalities for fractional integrals hold:

$$
\begin{aligned}
& \left|\frac{\Gamma(\alpha)}{(b-a)^{\alpha}}\left[J_{a^{+}}^{\alpha} f(b)+J_{b^{-}}^{\alpha} f(a)\right]-\frac{f\left(\frac{a+b}{2}\right)}{\alpha h\left(\frac{1}{2}\right)}\right| \\
& \quad \leq \frac{\Gamma(\alpha)}{(b-a)^{\alpha}}\left[J_{a^{+}}^{\alpha} g(b)+J_{b^{-}}^{\alpha} g(a)\right]-\frac{g\left(\frac{a+b}{2}\right)}{\alpha h\left(\frac{1}{2}\right)}
\end{aligned}
$$

and

$$
\begin{aligned}
& {[f(a)+f(b)]\left[\int_{0}^{1} t^{\alpha-1}(h(t)+h(1-t)) d t\right]} \\
& -\frac{\Gamma(\alpha)}{2(b-a)^{\alpha}}\left[J_{a^{+}}^{\alpha} f(b)+J_{b^{-}}^{\alpha} f(a)\right] \\
& \leq[g(a)+g(b)]\left[\int_{0}^{1} t^{\alpha-1}(h(t)+h(1-t)) d t\right] \\
& -\frac{\Gamma(\alpha)}{2(b-a)^{\alpha}}\left[J_{a^{+}}^{\alpha} g(b)+J_{b^{-}}^{\alpha} g(a)\right]
\end{aligned}
$$

with $\alpha \geq 0$.

Proof. In Definition 6, if we choose $\lambda=\frac{1}{2}$, we get

$$
\left|h\left(\frac{1}{2}\right)[f(x)+f(y)]-f\left(\frac{x+y}{2}\right)\right| \leq h\left(\frac{1}{2}\right)[g(x)+g(y)]-g\left(\frac{x+y}{2}\right)
$$

for all $x, y \in[a, b]$. Then if we take $x=t a+(1-t) b$ and $y=(1-t) a+t b$, we get

$$
\begin{aligned}
& \left|h\left(\frac{1}{2}\right)[f(t a+(1-t) b)+f((1-t) a+t b)]-f\left(\frac{a+b}{2}\right)\right| \\
& \quad \leq h\left(\frac{1}{2}\right)[g(t a+(1-t) b)+g((1-t) a+t b)]-g\left(\frac{a+b}{2}\right)
\end{aligned}
$$

for $t \in[0,1]$. Multiplying (2.3) by $t^{\alpha-1}$, then integrating the deduced inequality with respect to $t$ over $[0,1]$, we obtain;

$$
\begin{aligned}
& \mid h\left(\frac{1}{2}\right) {\left[\int_{0}^{1} t^{\alpha-1}(f(t a+(1-t) b)) d t+\int_{0}^{1} t^{\alpha-1}(f((1-t) a+t b)) d t\right] } \\
&-f\left(\frac{a+b}{2}\right) \int_{0}^{1} t^{\alpha-1} d t \mid
\end{aligned}
$$




$$
\begin{aligned}
\leq h & \left(\frac{1}{2}\right)\left[\int_{0}^{1} t^{\alpha-1}(g(t a+(1-t) b)) d t+\int_{0}^{1} t^{\alpha-1}(g((1-t) a+t b)) d t\right] \\
& -g\left(\frac{a+b}{2}\right) \int_{0}^{1} t^{\alpha-1} d t
\end{aligned}
$$

If we correct the above inequality, we get the first part of requested inequality.

$$
\begin{aligned}
& \left|\frac{\Gamma(\alpha)}{(b-a)^{\alpha}}\left[J_{a^{+}}^{\alpha} f(b)+J_{b^{-}}^{\alpha} f(a)\right]-\frac{f\left(\frac{a+b}{2}\right)}{\alpha h\left(\frac{1}{2}\right)}\right| \\
& \quad \leq \frac{\Gamma(\alpha)}{(b-a)^{\alpha}}\left[J_{a^{+}}^{\alpha} g(b)+J_{b^{-}}^{\alpha} g(a)\right]-\frac{g\left(\frac{a+b}{2}\right)}{\alpha h\left(\frac{1}{2}\right)} .
\end{aligned}
$$

To get second part of Theorem 10, let's use Definition 6. Then,

$$
\begin{aligned}
& |h(t) f(a)+h(1-t) f(b)-f(t a+(1-t) b)| \\
& \quad \leq h(t) g(a)+h(1-t) g(b)-g(t a+(1-t) b)
\end{aligned}
$$

and

$$
\begin{aligned}
& |h(1-t) f(a)+h(t) f(b)-f((1-t) a+t b)| \\
& \quad \leq h(1-t) g(a)+h(t) g(b)-g((1-t) a+t b) .
\end{aligned}
$$

Obtained last two inequalities if add side by side, we get

$$
\begin{aligned}
& {[f(a)+f(b)][h(t)+h(1-t)]-f(t a+(1-t) b)-f((1-t) a+t b)} \\
& \quad \leq[g(a)+g(b)][h(t)+h(1-t)]-g(t a+(1-t) b)-g((1-t) a+t b) .
\end{aligned}
$$

Multiplying the last inequality by $t^{\alpha-1}$, then integrating with respect to $t$ over $[0,1]$, we obtain;

$$
\begin{aligned}
{[f(a)+f(b)] } & {\left[\int_{0}^{1} t^{\alpha-1}(h(t)+h(1-t)) d t\right] } \\
& -\int_{0}^{1} t^{\alpha-1} f(t a+(1-t) b) d t-\int_{0}^{1} t^{\alpha-1} f((1-t) a+t b) d t \\
\leq & {[g(a)+g(b)]\left[\int_{0}^{1} t^{\alpha-1}(h(t)+h(1-t)) d t\right] } \\
& -\int_{0}^{1} t^{\alpha-1} g(t a+(1-t) b) d t-\int_{0}^{1} t^{\alpha-1} g((1-t) a+t b) d t .
\end{aligned}
$$

If we correct the obtained inequality, we get the desired inequality,

$$
\begin{array}{r}
{[f(a)+f(b)]\left[\int_{0}^{1} t^{\alpha-1}(h(t)+h(1-t)) d t\right]} \\
-\frac{\Gamma(\alpha)}{2(b-a)^{\alpha}}\left[J_{a^{+}}^{\alpha} f(b)+J_{b^{-}}^{\alpha} f(a)\right]
\end{array}
$$




$$
\begin{gathered}
\leq[g(a)+g(b)]\left[\int_{0}^{1} t^{\alpha-1}(h(t)+h(1-t)) d t\right] \\
-\frac{\Gamma(\alpha)}{2(b-a)^{\alpha}}\left[J_{a^{+}}^{\alpha} g(b)+J_{b^{-}}^{\alpha} g(a)\right] .
\end{gathered}
$$

So the proof is completed.

Corollary 1. If we choose $h(t)=t$ and $\alpha=1$ in Theorem 10, we get the results of Theorem 3.

Corollary 2. If we choose $h(t)=t$ in Theorem 10, we get the result of Theorem 7 . Also, if we choose $\alpha=1$, we obtain Theorem 5 .

Theorem 11. Let $f, g:[0, \infty) \rightarrow \mathbb{R}$ be positive functions with $0 \leq a<b$ and $f, g \in$ $L_{1}\left[a, \frac{b}{m}\right]$. If $g$ is an $m-$ convex function on $[0, \infty)$ and $f$ is a $(g, m)$ - convex dominated function, then the following inequalities for fractional integrals hold:

$$
\begin{aligned}
& \left|\frac{\Gamma(\alpha+1)}{2(b-a)^{\alpha}}\left[J_{a^{+}}^{\alpha} f(b)+m^{\alpha+1} J_{\frac{b}{m}}^{\alpha} f\left(\frac{a}{m}\right)\right]-f\left(\frac{a+b}{2}\right)\right| \\
& \quad \leq \frac{\Gamma(\alpha+1)}{2(b-a)^{\alpha}}\left[J_{a^{+}}^{\alpha} g(b)+m^{\alpha+1} J_{\frac{b}{m}}^{\alpha} g\left(\frac{a}{m}\right)\right]-g\left(\frac{a+b}{2}\right)
\end{aligned}
$$

and

$$
\begin{aligned}
\mid \frac{1}{2}[ & \left.\frac{\alpha f(a)+m f\left(\frac{a}{m}\right)}{\alpha+1}+m \frac{f\left(\frac{b}{m}\right)+m \alpha f\left(\frac{b}{m^{2}}\right)}{\alpha+1}\right] \\
& -\frac{\Gamma(\alpha+1)}{2(b-a)^{\alpha}}\left[J_{a^{+}}^{\alpha} f(b)+m^{\alpha+1} J_{\frac{b}{m}}^{\alpha} f\left(\frac{a}{m}\right)\right] \mid \\
\leq & \frac{1}{2}\left[\frac{\alpha g(a)+m g\left(\frac{a}{m}\right)}{\alpha+1}+m \frac{g\left(\frac{b}{m}\right)+m \alpha g\left(\frac{b}{m^{2}}\right)}{\alpha+1}\right] \\
& -\frac{\Gamma(\alpha+1)}{2(b-a)^{\alpha}}\left[J_{a^{+}}^{\alpha} g(b)+m^{\alpha+1} J_{\frac{b}{m}}^{\alpha}-g\left(\frac{a}{m}\right)\right]
\end{aligned}
$$

with $\alpha>0$ and $m \in(0,1]$.

Proof. In Definition 5, if we choose $\lambda=\frac{1}{2}$ and $x=t a+(1-t) b, y=(1-t) \frac{a}{m}+t \frac{b}{m}$, we get

$$
\begin{aligned}
& \left|\frac{f(t a+(1-t) b)+m f\left((1-t) \frac{a}{m}+t \frac{b}{m}\right)}{2}-f\left(\frac{a+b}{2}\right)\right| \\
& \quad \leq \frac{g(t a+(1-t) b)+m g\left((1-t) \frac{a}{m}+t \frac{b}{m}\right)}{2}-g\left(\frac{a+b}{2}\right) .
\end{aligned}
$$


Multiplying the last inequality by $t^{\alpha-1}$, then integrating with respect to $t$ over $[0,1]$, we obtain;

$$
\begin{aligned}
& \mid \frac{1}{2}\left[\int_{0}^{1} t^{\alpha-1}(f(t a+(1-t) b)) d t+m \int_{0}^{1} t^{\alpha-1} f\left((1-t) \frac{a}{m}+t \frac{b}{m} d t\right]\right. \\
& \quad-f\left(\frac{a+b}{2}\right) \int_{0}^{1} t^{\alpha-1} d t \mid \\
& \quad \leq \frac{1}{2}\left[\int_{0}^{1} t^{\alpha-1}(g(t a+(1-t) b)) d t+m \int_{0}^{1} t^{\alpha-1} g\left((1-t) \frac{a}{m}+t \frac{b}{m}\right) d t\right] \\
& \quad-g\left(\frac{a+b}{2}\right) \int_{0}^{1} t^{\alpha-1} d t .
\end{aligned}
$$

If the necessary calculations are made and the resulting expression is edited, the first part of Theorem 11 is obtained. To get second part of Theorem 11, we can use Definition 5. Then, if we choose $x=a$ and $y=\frac{b}{m}$, we get;

$$
\begin{aligned}
& \left|t f(a)+m(1-t) f\left(\frac{b}{m}\right)-f(t a+m(1-t)) \frac{b}{m}\right| \\
& \quad \leq t g(a)+m(1-t) g\left(\frac{b}{m}\right)-g\left(t a+m(1-t) \frac{b}{m}\right) .
\end{aligned}
$$

Also, in Definition 5 if we choose $x=\frac{a}{m}$ and $y=\frac{b}{m^{2}}$, then multiplying the obtained inequality with $m$, the following inequality is obtained.

$$
\begin{aligned}
\mid m t f & \left(\frac{a}{m}\right)+m^{2}(1-t) f\left(\frac{b}{m^{2}}\right)-m f\left(t \frac{a}{m}+m(1-t) \frac{b}{m^{2}}\right) \mid \\
& \leq m t g\left(\frac{a}{m}\right)+m^{2}(1-t) g\left(\frac{b}{m^{2}}\right)-m g\left(t \frac{a}{m}+m(1-t) \frac{b}{m^{2}}\right) .
\end{aligned}
$$

Let's multiply both of the last two inequalities we got above by $t^{\alpha-1}$,then integrate the obtained inequality with respect to $t$ over $[0,1]$,

$$
\begin{aligned}
& \left|\frac{f(a)}{\alpha+1}+m \frac{f\left(\frac{b}{m}\right)}{\alpha(\alpha+1)}-\frac{\Gamma(\alpha)}{(b-a)^{\alpha}} J_{a^{+}}^{\alpha} f(b)\right| \\
& \leq \frac{g(a)}{\alpha+1}+m \frac{g\left(\frac{b}{m}\right)}{\alpha(\alpha+1)}-\frac{\Gamma(\alpha)}{(b-a)^{\alpha}} J_{a^{+}}^{\alpha} g(b)
\end{aligned}
$$

and

$$
\left|m \frac{f\left(\frac{a}{m}\right)}{\alpha(\alpha+1)}+m^{2} \frac{f\left(\frac{b}{m^{2}}\right)}{\alpha+1}-m^{\alpha+1} \frac{\Gamma(\alpha)}{(b-a)^{\alpha}} J_{\frac{b}{m}}^{\alpha}-f\left(\frac{a}{m}\right)\right|
$$




$$
\leq m \frac{g\left(\frac{a}{m}\right)}{\alpha(\alpha+1)}+m^{2} \frac{g\left(\frac{b}{m^{2}}\right)}{\alpha+1}-m^{\alpha+1} \frac{\Gamma(\alpha)}{(b-a)^{\alpha}} J_{\frac{b}{m}}^{\alpha}-g\left(\frac{a}{m}\right) .
$$

Finally, the second part of the theorem is proved when we arrange the inequalities we obtained using the properties of absolute value. Thus, the proof is completed.

Corollary 3. If we choose $m=1$ and $\alpha=1$ in Theorem 11, we get the results of Theorem 3.

Corollary 4. If we choose $\alpha=1$ in Theorem 11, we get the results of Theorem 4 . Also, if we choose $m=1$ in Theorem 11, we get the result of Theorem 7.

\section{REFERENCES}

[1] S. Belarbi and Z. Dahmani, "On some new fractional integral inequalities," J. Ineq. Pure Appl. Math., vol. 10, p. Art. 86, 2009. [Online]. Available: https://www.emis.de/journals/JIPAM/ images/139_09_JIPAM/139_09.pdf

[2] Z. Dahmani, "New inequalities in fractional integrals," Int. J. Nonlinear Sci., vol. 9, pp. 493-497, 2010.

[3] M. Dokuyucu, "Caputo and Atangana-Baleanu-Caputo fractional derivative applied to garden equation," Turkish Journal of Science, vol. 5, pp. 1-7, 2020. [Online]. Available: https://dergipark.org.tr/en/download/article-file/1024808

[4] S. S. Dragomir, "On some new inequalities of Hermite-Hadamard type for $m$-convex functions," Tamkang Journal of Mathematics, vol. 33, no. 1, pp. 55-65, 2002, doi: 10.5556/j.tkjm.33.2002.304.

[5] S. S. Dragomir and N. M. Ionescu, "On some inequalities for convex-dominated functions," Anal. Num. Theor. Approx., vol. 19, pp. 21-28, 1990.

[6] S. S. Dragomir and C. E. M. Pearce, "Selected topics on Hermite-Hadamard inequalities and applications," RGMIA Monographs, 2000.

[7] S. Dragomir, C. Pearce, and J. Pecaric, "Means, g-convex dominated and Hadamard- type inequalities," Tamsui Oxford Journal of Mathematical Sciences, vol. 18, pp. 161-173, 2002.

[8] A. Ekinci and M. E. Ozdemir, "Some New Integral Inequalities Via Riemann Liouville Integral Operators," Applied and Computational Mathematics, vol. 3, pp. 288-295, 2019.

[9] R. Gorenflo and F. Mainardi, Fractional calculus: integral and differential equations of fractional order. Wien: Springer, 1997.

[10] M. Gürbüz and M. Özdemir, "On some inequalities for product of different kinds of convex functions," Turkish Journal of Science, vol. 5, pp. 23-27.

[11] A. Házy, "Bernstein-Doetsch type results for $h$-convex functions," Math. Inequal. Appl., vol. 14, no. 3, pp. 499-508, 2011, doi: 10.7153/mia-14-42.

[12] H. Kavurmaci, "Bazı farklı türden konveks fonksiyonlar icin Ostrowski ve Hermite-Hadamard tipli esitsizlikler," Ataturk University, Graduate School of Natural and Applied Sciences, 2012.

[13] K. S. Miller and B. Ross, An introduction to the Fractional Calculus and Fractional Differential Equations. John Wiley and Sons, 1993.

[14] M. E. Ozdemir and H. K. Onalan, "Hermite-Hadamard's inequalities for different kinds of convexity via fractional integrals," Submitted.

[15] J. E. Pecaric, F. Proschan, and Y. L. Tong, Convex Functions, Partial Orderings and Statistical Applications. Boston, MA etc.: Academic Press, 1992.

[16] I. Podlubny, Fractional Differential Equations. Academic Press, San Diego, 1999. 
[17] M. Z. Sarikaya and N. Alp, "On Hermite-Hadamard-Fejer type integral inequalities for generalized convex functions via local fractional integrals," Open Journal of Mathematical Sciences, vol. 3, pp. 273-284, 2019, doi: 10.30538/oms2019.0070.

[18] M. Z. Sarikaya, A. Saglam, and H. Yildirim, "On some Hadamard-type inequalities for $h$-convex functions,” J. Math. Inequal., vol. 2, pp. 335-341, 2008, doi: 10.7153/jmi-02-30.

[19] M. Z. Sarikaya, E. Set, H. Yaldiz, and N. Basak, "Hermite-Hadamard's inequalities for fractional integrals and related fractional inequalities," Mathematical and Computer Modelling, vol. 57, pp. 2403-2407, 2013, doi: 10.1016/j.mcm.2011.12.048.

[20] E. Set, A. Akdemir, and E. Alan, "Hermite-Hadamard and Hermite-Hadamard-Fejer type inequalities involving fractional integral operators," Filomat, vol. 33, no. 8, pp. 2367-2380, 2019, doi: 10.2298/FIL1908367S.

[21] E. Set, A. Akdemir, and M. Özdemir, "Simpson type integral inequalities for convex functions via Riemann-Liouville integrals," Filomat, vol. 31, no. 14, pp. 4415-4420, 2017, doi: 10.2298/FIL1714415S.

[22] G. Toader, "Some generalizations of the convexity," Proc. Colloq. Approx. Optim. Univ, pp. 329$338,1984$.

[23] S. Varosanec, “On h-convexity,” J. Math. Anal. Appl., vol. 326, pp. 303-311, 2007, doi: 10.1016/j.jmaa.2006.02.086.

[24] F. Yalçın and N. Bekar, "Two-dimensional operator harmonically convex functions and related generalized inequalities,” Turkish Journal of Science, vol. 4, pp. 30-38.

[25] C. Yildiz, M. E. Ozdemir, and H. K. Onalan, "Fractional integral inequalities for different functions," New Trends in Mathematical Sciences, vol. 3, pp. 110-117, 2015. [Online]. Available: https://www.ntmsci.com/AjaxTool/GetArticleByPublishedArticleId?PublishedArticleId=66

Author's address

Havva Kavurmaci-Önalan

Van Yüzüncü Yil University, Faculty of Education, Department of Mathematics Education, Van, Turkey

E-mail address: havvaonalan@yyu.edu.tr 